\title{
Task-Specific Knowledge of the Law of Pendulum Motion in Children and Adults
}

Andrea Frick, Susanne Huber, Ulf-Dietrich Reips, and Horst Krist

University of Zürich, Switzerland

The present experiment investigated children and adults' knowledge of the pendulum law under different task conditions. The question asked was whether adults and fourth-graders knew that the period of a pendulum is a function of pendulum length but is independent of its mass. The task was to judge the period on a rating scale (judgment task), to imagine the swinging pendulum and indicate the corresponding time interval (imagery task), or to adjust the period of a dynamically presented pendulum (perception task). Normative consideration of pendulum length as the only relevant factor was primarily found in the perception task and, for adults, in the imagery task, whereas in the judgment task, children and adults frequently considered the irrelevant dimension of mass. Most children showed poor imagery performance. Preceding adjustment (perception task) and rating (judgment task) had no differential influence on subsequent imagery performance.

Keywords: Intuitive knowledge, physics, pendulum, cognitive development, children, imagery

Research in the field of intuitive physics suggests that people have some kind of intuitive knowledge about physical events they encounter in everyday life. We are, for example, able to judge the distance of objects solely by motion that is subject to physical forces like gravity (Hecht, Kaiser, \& Banks, 1996; Watson, Banks, von Hofsten, \& Royden, 1992), to perceive violations of mechanical principles in dynamical displays (Shanon, 1976; Spelke, Breinlinger, Macomber, \& Jacobson, 1992), and to recognize even minor manipulations of tempo in video play backs of human motion (Johansson \& Jansson, 1967). These and many other findings show that although we may not know the exact formula or rule that describes a movement, in many cases we are quite sensitive to violations of the underlying mechanical principles.

Alongside these astonishing perceptual competencies, however, people hold erroneous beliefs about motion, inconsistent with the laws of Newtonian physics. Although the mechanical principles governing such motions have been known for centuries, even well-educated adults seem to believe that objects behave differently (Clement, 1982;
Krist, Fieberg, \& Wilkening, 1993; McCloskey, 1983; McCloskey, Caramazza, \& Green, 1980).

People's intuitive physics seems to be highly task-specific, and considerable performance dissociations can be found in children and adults (Wilkening \& Huber, 2002). In abstract judgment tasks, children and often adults tend to apply simple heuristics such as focusing on one dimension (Piaget, 1970), or integrating the relevant dimensions in an additive manner (Anderson, 1980; Wilkening, 1980). On the other hand, they frequently show normative integration strategies in tasks that assess their motor competencies, which they have adapted to the laws of motion through everyday experience (Huber, Krist, \& Wilkening, 2003; Krist et al., 1993; Krist, Loskill, \& Schwarz, 1996; Wilkening, 1980, 1981). Similarly, in perceptual tasks based on distinguishing normative and anomalous dynamics when viewing ongoing events, children and adults tend to perform better than in static judgment tasks (Kaiser, Proffitt, 1984; Kaiser, Proffitt, \& Anderson, 1985; Kim \& Spelke, 1999). These findings suggest that different knowledge formats or mental rep- 
resentations are activated depending on how abstract the task is. However, it is not only action tasks or perceptual tasks that can cause people to exhibit adequate knowledge. Studies on mental imagery have shown that even simulated action or viewing (Huber \& Krist, 2004; Schwartz \& Black, 1999) can improve participants' performance tremendously. One possible explanation for this improvement is that perceptual or perceptual-motor representations are activated when imagining an event.

It is widely agreed that mental images and representations arising from normal visual perception have much in common. There is psychophysical evidence that neural mechanisms generally identified with early stages of visual processing are engaged in imagery (Gilden, Blake, \& Hurst, 1995). Results from neuro-imaging studies also yield support for the assumption that imagery and perception are closely related processes. It has been shown that some brain areas activated by imagery are also active during perception (Kosslyn, Thompson, Kim, \& Alpert, 1995). Thus, one could reasonably expect that performance in a perceptual task, and in one where participants have to imagine the same event, would follow similar principles. Does this mean that people's mental images are not influenced by their beliefs? If people hold erroneous beliefs about a physical event that are revealed in an abstract judgment task, will these beliefs influence the way they would mentally simulate the event?

Schwartz (1999) took a closer look at the interference of people's beliefs with their imagery ability in the domain of motor imagery. In his experiment participants had to simulate the tilting of glasses of different diameters so that the imagined water level reached the rim. His results showed that when the participants had to simulate a tilt as a first task, they correctly tilted the thin glass farther than the wide one. In contrast, when they completed the simulation task after a judgment task, participants' initial judgments interfered with their subsequent tilts. These tilts were not only incorrect, but also did not in fact represent the beliefs of the participants. According to Schwartz, the preceding explicit judgment task (i.e., the activation of people's beliefs about the spatial outcome) undermined the participants' ability to complete the task through imagery. In another experiment, Schwartz found that dynamic perceptual information (e.g., torque) and beliefs about physical properties (e.g., viscosity) affect the inferences adults draw through imagery.

Few would deny that imagery can be modified or influenced by cognitive processes. However, there is still the question as to what extent it can be influenced, and of how far the equivalence in imagery and perception goes. There is evidence that functional equivalence in imagery and perception can extend to levels of the visual system where the effects produced cannot be explained by what people know about objects and events, or by how they expect them to perform (Finke, 1980).

The question of whether and to what extent imagery is influenced by beliefs may best be addressed by investigating situations in which people's beliefs differ from what they perceive when observing an event. In an experiment investigating whether prism-induced pointing aftereffects could be evoked by imagery as well as by perception, Finke (1979) led participants to expect aftereffects opposite to those produced. He found that, even if contrary to expectations, mental images still determined the direction of pointing aftereffects.

Instead of inducing false expectations by instruction, one could also use topics for which it is reasonable to suppose that false expectations already exist. Thus, topics for which false beliefs and task dissociations can be found seem suitable for this purpose. Task dissociations have been studied, mainly in the field of cognitive development, in order to learn about children's knowledge acquisition. An important question is whether perceptual, perceptualmotor, or even imagery abilities may serve as precursors to more formal and abstract knowledge, or whether these knowledge formats develop independently from each other. Although in recent years a large amount of literature in the field of cognitive development has been concerned with these questions, the origins of many misconceptions and task dissociations, and the nature of the underlying representations, are far from being clear.

The present experiment tries to address these questions about the nature of children and adults' representations, by using three different task formats in order to assess their performance in a perceptual, an imagery, and a more abstract judgment task. The motion of a physical pendulum was chosen as the problem instance, as some task dissociations could be expected on the basis of earlier studies. Below, we will first review the most relevant of these earlier studies; this will be followed by a description of the specifics of our own experiment.

Pendulum motion is something we can observe in daily life, in the form of swings, pendulum clocks, dangling objects, and the like. Former pendulum studies have shown that adults are quite good at recognizing the normative relationship between pendulum length and period (see Equation 1), when actually viewing a swinging pendulum. The first to report on this perceptual competence was Paolo Bozzi (1958). By means of a mechanical apparatus, he investigated which periods the participants rated as correct for pendulums of differing length $(10 \mathrm{~cm}, 20 \mathrm{~cm}$, and 40 $\mathrm{cm})$. Bozzi found that, as the length of the pendulum increased, the rated periods had higher values, which is in line with the pendulum law. But these subjectively natural periods were longer than those an identical pendulum would exhibit while swinging freely. 
$T=2 \pi \sqrt{L / g}$

where $\quad T=$ time to swing through one complete cycle (period);

$L=$ distance from the fulcrum to the center of the bob;

$g=$ gravitational acceleration

Pittenger (1990) studied perceived naturalness of pendulum motion by means of a manipulable apparatus. As in Bozzi's (1958) experiment, the period of the pendulum could be varied independently of its length and angle of deflection. The naturalness ratings showed a high sensitivity to violations of the length-period relation, and even asymmetries in the oscillation (caused by technical imperfections) were discerned. Again, the pendulum motions perceived as natural were somewhat slower than the normative motions.

In short, these findings show that we are very responsive to violations of pendulum motion, but there is a persistant bias. In accordance with the studies on intuitive physics reported above, discrepancies have been noted (Bozzi, 1958; Pittenger, 1985) between participants' performances in perception-based tasks and their explicit verbalizations. Unfortunately, these dissociations have never been studied in more detail. So, one question addressed in this experiment was whether a dissociation can be found between participants' appraisals of period in a perception task and a more abstract yet nonverbal task.

Although Newtonian physics shows that pendulum length is the only relevant factor that has a significant influence on period, people might hold different beliefs: there might be other aspects of a pendulum than its length that might influence its swinging behavior. Until now, the role of pendulum mass in period estimations has not been studied systematically. One reason why pendulum mass has often been disregarded in research might be that it is physically irrelevant. Nevertheless, it might be of psychological relevance, especially since it seems rather counterintuitive to many people that pendulum mass does not affect the period. In fact, Galileo Galilei faced quite some resistance when trying to convince his contemporaries of his findings about the independence of pendulum period from pendulum mass (for a historical digest see Bozzi, 1958). Bozzi also reported that some of his own participants, when questioned about their convictions on the physical motion of pendulums, would claim that "the heavier a pendulum is, the slower it moves" (on the grounds that it is more massive and thus encounters more inertia). Some other participants, however, would argue that "the more it weighs, the faster it moves" (by analogy to the erroneous assumption that its weight makes it fall more rapidly).
Apparently, there are some uncertainties about the role of pendulum mass and people seem to apply divergent heuristics when they have to verbalize the relation explicitly. In the present experiment, it was investigated whether changes in the length and in the apparent mass of the pendulum would affect nonverbal responses (i.e., ratings or adjustments of the pendulum period). The questions addressed were, what do people know about the relatedness of pendulum length to pendulum period, and pendulum mass to pendulum period, and whether they show any erroneous beliefs about either of these relationships.

To address the question of what children know of the pendulum law at an age when they have had no formal education about the specific problem at school, 8 to 10 yearold fourth-graders were tested. As is the case for adults, previous studies on children's knowledge of the pendulum law only focused on the length-period relation. Spillane, Turcotte, and Pufall (1991) investigated children's knowledge of the relation between pendulum length and period using Pittenger's (1985) method. The children's task was to predict the length of the pendulum from the motion of the swinging pendulum. The authors found that a qualitative developmental change occurs between age 6 and 9: from not discriminating the positive functional relation between length and period, to discriminating their ordinal relation. Older age groups, 11 and 18 yearolds, showed a mere quantitative refinement of this discrimination.

In light of Spillane et al.'s (1991) findings, some basic knowledge of the length-period relationship could be expected in fourth-graders; thus we expected children to perform well in a perception-based task. However, it was debatable whether and how children would consider the factors of length and pendulum mass in a more abstract task. Furthermore, if the expected dissociation between abstract and perception-based tasks could be found in children and/or adults, how participants imagined the pendulum motion would be of particular interest.

In previous research, several approaches to assessing people's intuitive knowledge have been used. One way is to let people infer one physical property on the basis of one or several other properties (e.g., Pittenger, 1985). Another approach is to find out whether or when people detect violations of physical laws, or how they judge the "naturalness" of a given process (e.g., Pittenger, 1990). Forced-choice paradigms are often used to assess whether participants can distinguish between correct and anomalous processes or scenes (e.g., Kaiser et al., 1985). Yet another way is to provide the opportunity to create or restore the correct physical proportions in "alternative realities" in which variables can be varied independently.

In the present experiment, a functional measurement 
method, based on information integration theory (Anderson, 1981, 1982), was applied, allowing participants to actively produce or recognize correct processes, rather than just telling two given versions apart. The stimuli were presented in virtual reality on a computer that simulated the physical properties of a pendulum in a real room. This method had the advantage that all variables could be manipulated separately, but it had the disadvantage that all objects had to be scaled to fit on the screen. Although we are quite used to this scaling from experience with television, it was nevertheless unclear how this scaling might affect estimations of naturalness.

In summary, the present experiment focuses on the acquisition of knowledge of pendulum motion. More specifically, we were interested in whether children and adults know that the period of a swinging pendulum depends on the length of the pendulum but not on its mass. A simulated pendulum, which varied in length and apparent bob mass, was presented in a virtual three-dimensional room on a computer screen. One major aim of the experiment was to understand both the processes active during imagery and perception of an ongoing event (i.e., the swinging pendulum), and the processes active during reasoning about the same event. Therefore task performance was studied in three task formats: Participants either conducted (i) an abstract judgment task where they had to indicate the period of the statically presented pendulum; or (ii) a perception-based task where they adjusted the swinging period of a dynamically presented pendulum such that the swing looked natural; or (iii) a visual imagery task where they imagined the statically presented pendulum to swing for three cycles.

The question was whether misbeliefs that show in the judgment task would also be present in the imagery task, and whether performance in the imagery task would be equivalent to performance in the perception task. If misbeliefs could be demonstrated in an abstract judgment task, but not in the perception-based task nor in an imagery task, this would be of considerable theoretical interest, because the imagined event would be independent of participants' beliefs about pendulum motion. Instead, it would corroborate the assumption of information-processing mechanisms that are shared by both imagery and perception and that are not influenced by how events might be conceptualized.

In order to study the influence of preceding reasoning processes and/or perceptual processes on subsequent visual imagery performance, all three participant groups conducted the imagery task in a second part of the experiment.

\section{Method}

\section{Participants}

Two age groups were tested. The adult participant group (24 male and 24 female) largely consisted of psychology students. Their age ranged from 20;0 (= 20 years and 0 months) to $60 ; 0$ (mean age $=26 ; 8$ ). A second group of participants consisted of 48 fourth-graders (24 male and 24 female; range $=8 ; 10$ to $10 ; 7$; mean age $=9 ; 10)$, who were tested in a suitable room at their school. Parents, school officials, and teachers were asked for their permission, and participation was completely voluntary. Participants were not paid or remunerated otherwise for their participation. The data of one child and one adult had to be replaced due to experimenter error.

\section{Stimuli}

Participants were shown a three-dimensional computer graphic of a virtual room (see Figure 1) on a 21 inch CRT (cathode ray tube) monitor. At a distance of $40 \mathrm{~cm}$ from the monitor, the extent of the graphic amounted to a visual angle of 33 degrees in width and 22 degrees in height. The side walls of the virtual room were partly displayed, in order to induce a stronger three-dimensional impression. A door and a window were displayed on the back wall to provide size reference. The door in the virtual room had an image size of $7 \mathrm{~cm}$ by $3.2 \mathrm{~cm}$, which corresponded to the size of a real door of approximately $200 \mathrm{~cm}$ in height and $90 \mathrm{~cm}$ in width. Thus, the proportion of the virtual to the real room was about 1:28, and one meter in the virtual reality amounted to a visual angle of about 5 degrees. A pendulum (i.e. a gray line with an orange cube at its lower end) was displayed attached to the rear wall. The pendulum was described as "a string with a brick attached to its lower end". A cube shaped bob was chosen in order

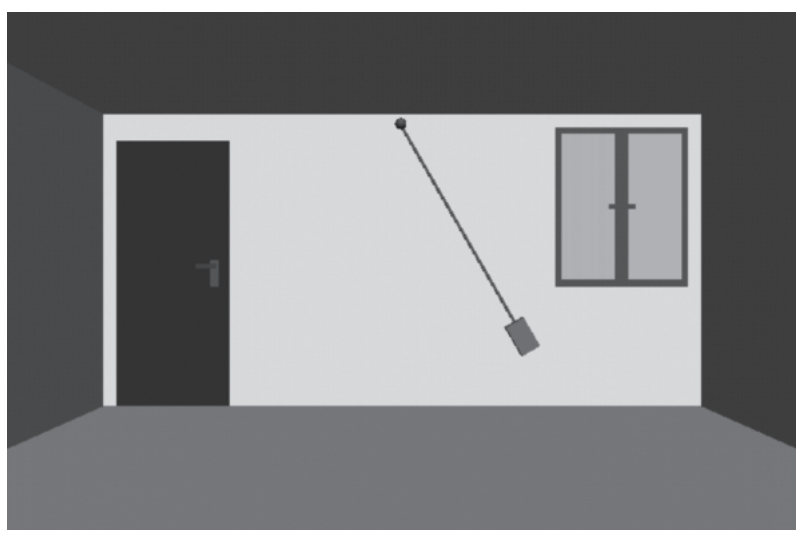

Figure 1. Computer graphic of the virtual room with a pendulum attached to the rear wall. 
to ensure that the apparent mass of the object could be varied without affecting the length of the pendulum, by varying the width of the cube ${ }^{1}$. The length and apparent mass of each pendulum (i.e. the size of the bob) were varied on three levels. The simulated lengths of the pendulums (i.e. the distance from the fulcrum to the center of the bob) corresponded to a real length of $60 \mathrm{~cm}, 120 \mathrm{~cm}$, and $180 \mathrm{~cm}$, respectively. These lengths correspond to swinging periods of 1.55, 2.20, and 2.69 seconds. The cube-shaped bob varied in width only, corresponding to $15 \mathrm{~cm}, 30 \mathrm{~cm}$, and $45 \mathrm{~cm}$. The pendulum was initially presented at a $30^{\circ} \mathrm{de}-$ flection angle (counterclockwise off the gravitational orientation) so that on release it would swing in the frontoparallel plane.

\section{Design}

Participants were assigned to one of three groups of 16 adults and 16 children ( 8 male and 8 female each). Assignment was random with the constraint of equal gender distribution. In the first part of the experiment, each group either completed a judgment task, a perception task, or an imagery task. In the second part of the experiment, all participants completed the imagery task. According to a complete factorial within-subjects design, every combination of length and mass was shown twice, resulting in 18 trials (two blocks of nine combinations). The experiment thus comprised a 2 (age group) $\times 3$ (task) $\times 2$ (part) $\times 2$ (block) $\times 3$ (length) $\times 3$ (mass) design, with age group and task as between-subjects factors, and part, block, length, and mass as within-subjects factors.

\section{General Procedure}

In the following, we will report on the common features of the three tasks and the general procedure, before we go into the peculiarities of the three tasks.

A female experimenter tested each participant individually. Participants were first asked to provide some demographic information, including gender, age, and nationality, and were then provided with the written instructions for the task. In order to make sure the children understood the task, the instructions were also read to them. The participants' task was to estimate the period of different pendulums. Participants received one of two trial orders that were predefined by a randomizing com-

1 By using a cube-shaped bob that varied in width but not in height or depth it was also possible to keep constant the side surface and thus the air resistance that such an object would encounter in the real world. Even if the air resistance of the bob did affect the responses, this would be comparable for all weights - except for some minor differences in air turbulence. puter program ${ }^{2}$. By means of three preceding practice trials, the experimenter could verify that the participants had understood the instructions. For the practice trials, combinations of length/bob size were used in the following order: $180 \mathrm{~cm} / 15 \mathrm{~cm}$ (see Figure 1), $180 \mathrm{~cm} / 45 \mathrm{~cm}, 60 \mathrm{~cm} /$ $45 \mathrm{~cm}$ (in trial order one); $60 \mathrm{~cm} / 45 \mathrm{~cm}, 60 \mathrm{~cm} / 15 \mathrm{~cm}$, $180 \mathrm{~cm} / 15 \mathrm{~cm}$ (in trial order two). Thus, participants knew the whole range of variation and could calibrate their answers accordingly. No feedback about the accuracy of the responses was given. At the end of the experiment, the participants were asked explicitly whether they applied a certain strategy, used a "trick", or thought in a particular way in order to complete the task. An experimental session took about 15 minutes.

\section{Judgment Task}

The pendulum was presented statically (as seen in Figure 1). The participants were instructed to judge on a rating scale how much time the pendulum would take to swing back and forth 50 times. This high number of cycles was chosen to make the strategy of imagining the swings less feasible than in the imagery task. The rating scale did not contain any objective reference points or digits. Participants were instructed that a mouse click on the right end of the scale indicated a very long time and a click on the left end of the scale stood for a very short amount of time. In order to visualize and sustain this instruction, pictures of a full and a near-empty sandglass were shown to the right and the left of the rating scale, respectively.

\section{Imagery Task}

The pendulum was presented statically and the participants were instructed to imagine the pendulum swinging three full cycles and to hold down the mouse button accordingly. The time the button was pressed down was recorded in milliseconds. The participants were asked to release the button exactly at the moment when they imagined the pendulum to arrive back at its initial position. Children especially sometimes double-clicked the button instead of keeping it pressed, or remarked that they had released the button unintentionally. In this case the corresponding trial was repeated.

\section{Perception Task}

The pendulum was presented dynamically. Initially, the pendulum would swing at a physically incorrect speed

2 This program follows the constraints that every combination is shown once in every block and no factor levels are repeated successively. 
$(10 \%, 20 \%$, or $30 \%$ too slow or too fast). The participants' task was to "find the correct speed" by adjusting the speed with the arrow keys of the computer keyboard, so that the motion looked "most natural". They were allowed to take as much time as they needed.

\section{Results}

In the following section, we will report the results from the first part of the experiment. This part consisted of the three different tasks and is therefore most relevant to our main questions. Results from the second part, in which all participants performed in the imagery task, will be reported subsequently, followed by analyses on a group level regarding the consistencies and absolute errors of the estimations.

\section{Individual Analyses - Part 1}

As mentioned in the introduction, Bozzi (1958) found diverging heuristics in the consideration of mass (the heavier pendulum swings slower, vs. the heavier pendulum swings faster). In order not to override such opposite effects by averaging, we analyzed each participant's data individually. As the method of functional measurement was used in this experiment, which implements a within-subjects design, an analysis of variance (ANOVA) could be calculated for every participant separately. On the basis of the factors that reach statistical significance in these individual ANOVAs (length $\times$ mass), one can infer which dimensions influenced each participant's responses regarding the period. Accordingly, participants were categorized into one of four groups that covered four theoretically ex- pected alternative strategies: the integration of both pendulum length and mass (main effects of both length and mass); the consideration of length only (single main effect of length); the consideration of mass only (single main effect of mass); and no consideration of any of the manipulated dimensions (no statistically significant main effects for neither of the two factors). Figure 2 shows the number of the applied strategies resulting from the individual ANOVAs for the first part of the experiment. The significance level was set at $10 \%$, to reduce the possibility of a beta-error (i.e., to reduce the risk of overlooking that a participant did consider a dimension).

The perception task yielded the best overall performance: out of a total of 32 participants (16 in each age group), 15 children considered the pendulum length exclusively, and 12 adults applied this normative strategy. In the imagery task, 11 children did not show any perspicuous strategy. Only 3 children considered the physically correct factor of length. However, adults performed just as well in the imagery task as in the perception task: all but one adult considered length exclusively. In the judgment task, an equal number of children considered the length of the pendulum as considered its mass (5 each). Only one child considered both dimensions, and 5 children considered neither mass nor length. In this task, none of the adults focused on mass exclusively, but 6 adults integrated both dimensions. Thus, although most children and adults applied the correct strategy in the perception task, some of them erroneously considered the pendulum mass in the judgment task. Children did this by focusing on pendulum mass exclusively, adults by integrating the mass and length information.

As expected, the manipulation of pendulum mass had divergent effects. Some participants considered the heavier bobs as swinging slower, and some participants con-

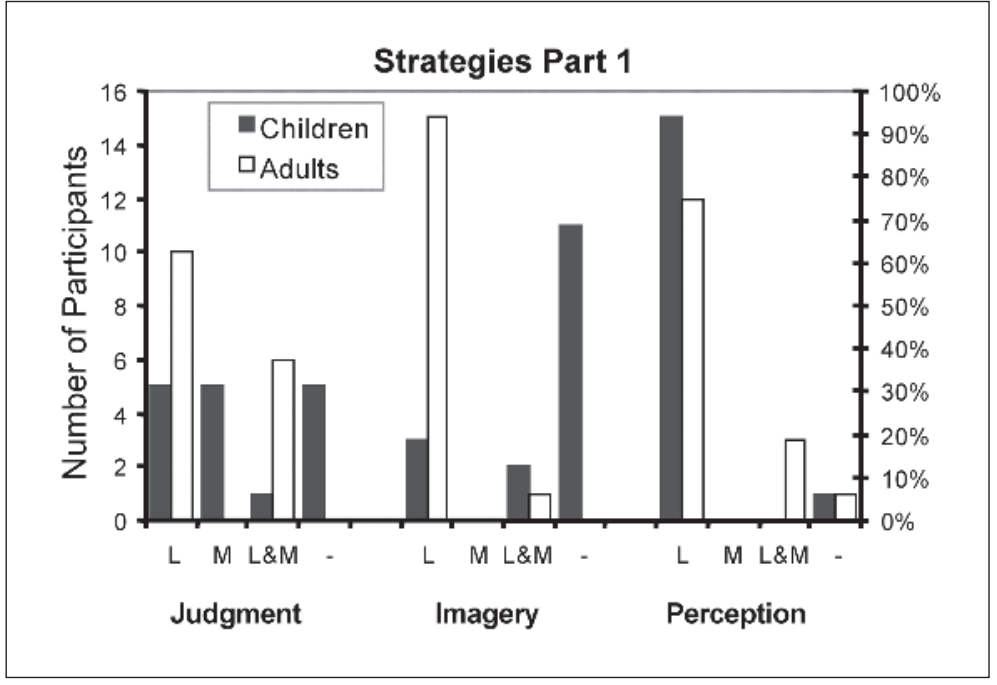

Figure 2. Number of the individually applied strategies (statistically significant factors in the ANOVAs, $p<.10$ ) for two age groups and three tasks. $\mathrm{L}=$ length; $\mathrm{M}=$ mass; $\mathrm{L} \& \mathrm{M}=$ length and mass; - = none. 
sidered them as swinging faster. Participants who considered the mass factor $(p<.10)$ were categorized as applying the "inertia strategy" if the marginal means of the estimated periods, collapsed over length, increased with increasing apparent mass (the heavier - the slower). Conversely, participants were categorized as applying the "gravity strategy", if the marginal means of the estimated periods decreased with increasing apparent mass (the heavier - the faster $)^{3}$. The "inertia strategy" was applied more frequently ( 6 children, 6 adults) than the "gravity strategy" (1 child, 3 adults). The rest were ambiguous (non-monotonic relations). No differing strategies could be found regarding the consideration of pendulum length. Longer pendulums were consistently rated as swinging slower.

\section{Individual Analyses - Part 2}

Participants' performance in the imagery task in part 2 of the experiment is displayed in Figure 3. Their superior performance in the perception task in part 1, compared to the judgment task, had no facilitating effect on the subsequent imagery performance. Neither children nor adults showed any remarkable differences in the subsequent imagery tasks in part 2. There was a slight drop in adults' performance in the imagery task in part 2 (all groups, Figure 3), compared to the imagery task in part 1 (middle group, Fig-

3 These category labels were derived from verbal reports of adult participants. By categorizing participants according to these labels, the authors do not want to imply that they all adopted this exact way of thinking. Their responses did fit with the concepts of gravity or inertia, but other strategies or other concepts (like resistance for example; see Bozzi 1958) might have led to the same pattern. ure 2). Children, on the other hand, showed slightly more length-only effects in the imagery tasks in part 2, than in the imagery task in part 1.

\section{Verbal Reports}

At the end of the experiment the participants were asked whether they applied a particular strategy. In the group that had completed the perception task, 1 child and 10 adults reported that they had considered both length and mass. In the group that had completed the judgment task, 3 children and 14 adults mentioned both dimensions. However, in the group that had completed the imagery task only, children and adults hardly ever mentioned the manipulated dimensions explicitly (children: once; adults: four times). Strategies like following the pendulum with one's eyes or head, counting, or intuitive guessing were predominantly reported here. Overall, only 2 children and 11 adults ( 7 times following the imagery task) mentioned the length factor exclusively. The mass factor was only infrequently mentioned on its own (children: four times - all following the judgment task; adults: three times). Analogies to swinging objects, like a pendulum clock or a seesaw, were most often reported after the perception task (children: twice; adults: three times). Overall, most explicit statements about length or mass were made after the judgment task, second most after the perception task, and fewest after the imagery task.

Participants' verbal reports did not reflect their responses in the three tasks very closely. Out of the 15 children and 12 adults who considered length in the perception task, none of the children and only 2 adults reported having considered this factor. Conversely, out of the 10 adults who did report having considered both factors in the perception task, only 1 was statistically significant.

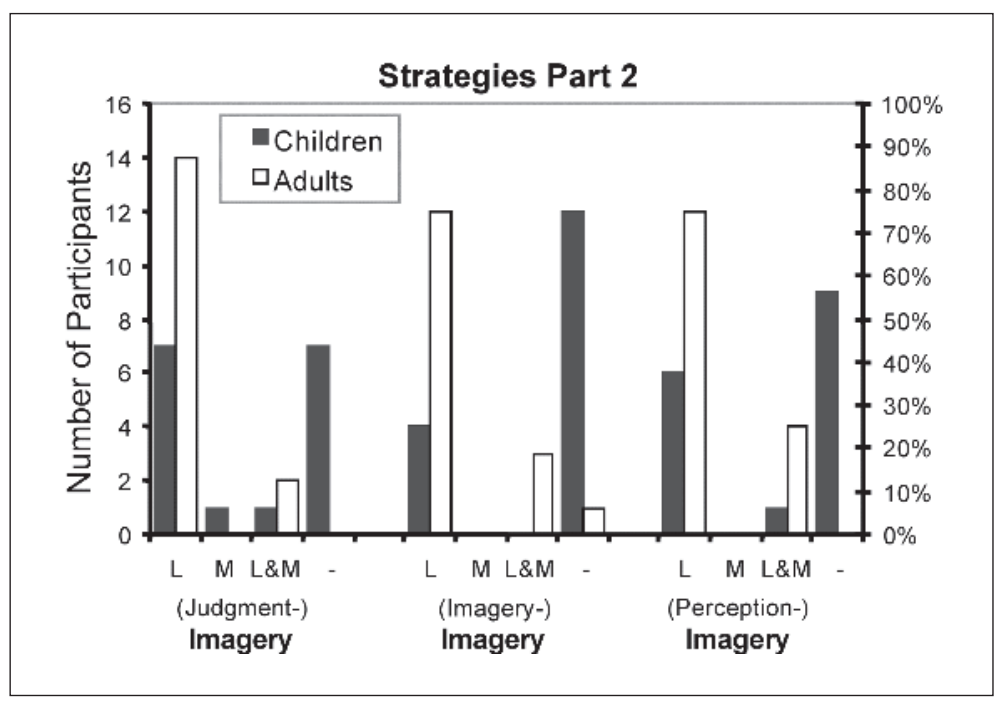

Figure 3. Number of the individually applied strategies (statistically significant factors in the ANOVAs, $p<.10$ ) for two age groups in the imagery tasks following the three tasks. $\mathrm{L}=$ length; $\mathrm{M}=$ mass; $\mathrm{L} \& \mathrm{M}=$ length and mass; - = none. 
Overall, for only 3 children and 19 adults ( $=23 \%$ of the total sample) were verbal reports consistent with responses.

\section{Consistencies}

For each participant and each task, the consistency of the responses was assessed by calculating the correlation (Pearson's $r$ ) between the two blocks of trials across the nine length-mass combinations. For statistical analyses, the resulting coefficients were converted to values of Fisher's $Z$ (also known as $z$ '). Table 1 shows the mean values for each age group and task in part 1 and part 2 .

To test whether the consistencies in the three conditions and the two age groups differed significantly, a univariate ANOVA, task (3) $\times$ age (2), was calculated with the consistencies in part 1 ( $Z$-values) as the dependent variable. The different tasks had a statistically significant effect on the consistencies, $F(2,90)=10.12, p<.001$. The adults showed equally good consistencies in the imagery task and in the perception task, but in the judgment task they responded much more consistently. Likewise, the children showed the highest consistencies in the judgment task. The age factor had a statistically significant effect on the consistencies, $F(1,90)=44.62, p<.001$. The children showed much lower overall consistencies than the adults. The interaction between the two factors of task and age did not reach statistical significance, $F(2,90)=3.6, p>.10$. Because children's consistencies were very low in the imagery tasks, the individual data resulting from these tasks lack statistical power and should be treated with caution.

The lower part of Table 1 shows the mean consistencies in the imagery tasks in part 2 , after completing either the perception task, the imagery task, or the judgment task in part 1 . Compared to the mean consistencies in the imagery task in part 1 (children: $r=.29$, adults: $r=.76$ ), the consistencies in the imagery tasks in part 2 were about the same or higher for adults. Children's mean consistencies in part 2 were higher if preceded by perception, and lower if preceded by imagery or judgment.

To test whether the consistencies in the imagery task of part 2 differed due to the task performed in part 1, an univariate ANOVA, task in part 1 (3) $\times$ age (2), was calculated with the consistencies in part 2 (Z-values) as dependent variable. The analysis yielded no statistically significant effect of the task in part $1, F(2,90)=1.11, p$ $>.33$. Thus, the first task (perception, judgment, or imagery) did not exert any influence on the consistencies in part 2. As in part 1, the age factor had a statistically significant main effect on the consistencies in part 2, $F(1,90)$ $=67.18, p<.001$, and no interaction between the task in part 1 and age was found, $F<1$.

\section{Error Analysis}

Our main interest did not concern the absolute accuracy but the relative gradations of the answers caused by the manipulated factors. Nevertheless, to obtain a measure for the mean absolute accuracy of the estimations, the mean constant error was calculated for the imagery and perception tasks by subtracting the norm values from the participants' responses. Accordingly, negative values stand for a predominant underestimation of the swinging period of the pendulum, and positive values stand for an overestimation of this period. Table 2 shows the mean deviations

Table 2

Mean Constant Errors and Standard Deviation, in Seconds per Cycle, for the Two Age Groups in the Imagery and Perception Task.

\begin{tabular}{lcccc}
\hline & \multicolumn{2}{c}{ Children } & \multicolumn{2}{c}{ Adults } \\
\cline { 2 - 5 } & $M$ & $S D$ & $M$ & $S D$ \\
\hline Imagery Task & -.156 & .718 & -.090 & .718 \\
Perception Task & -.024 & .529 & .353 & .636 \\
\hline
\end{tabular}

Table 1

Mean Consistencies (Correlations between Block 1 and Block 2): Pearson's r. Fisher's Z, and Standard Deviation of Fischer's Z.

\begin{tabular}{lcccccc}
\hline & \multicolumn{3}{c}{ Children } & \multicolumn{3}{c}{ Adults } \\
\cline { 2 - 8 } & $r$ & $Z$ & $S D$ & $r$ & $Z$ & $S D$ \\
\hline Part 1 & & & & & \\
Judgment Task & .61 & 0.70 & 0.87 & .94 & 1.72 & 0.51 \\
Imagery Task & .29 & 0.30 & 0.59 & .76 & 0.99 & 0.33 \\
Perception Task & .47 & 0.51 & 0.34 & .76 & 0.99 & 0.35 \\
\hline Part 2 & & & & & & \\
(Judgment-) Imagery Task & .12 & 0.12 & 0.56 & .85 & 0.85 & 0.56 \\
(Imagery-) Imagery Task & .11 & 0.11 & 0.46 & .78 & 1.05 & 0.65 \\
(Perception-) Imagery Task & .39 & 0.41 & 0.65 & .82 & 1.16 & 0.44 \\
\hline
\end{tabular}


from the physical norm in the perception and the imagery task for each age group. For the judgment task, no objective norm could be determined due to the personalized and thus relative nature of the rating scale.

The mean constant error in the imagery task was slightly higher for children than for adults. In the perception task, however, the mean constant error was smaller for the children. In the perception task, for a pendulum motion to "seem natural", adults chose a period that was on average 0.35 seconds (i.e., $16 \%$ of the average normative period) too long. However, this overestimation of the period was not found in the imagery task or in the children's perception task. Here the period was more likely to be underestimated, but was, on average, much closer to the physical norm. However, the standard deviation in all age groups was very high, and neither age, $F(1,60)=1.82, p>.05$, nor task, $F(1,60)=3.08, p>.05$, nor the interaction age $\times$ task, $F<1$, had statistically significant effects.

\section{Discussion}

As expected, there were considerable differences in performance between the three tasks. Both children and adults showed misbeliefs about the role of the pendulum mass in the judgment task. This hardly occurred in the perception task. Here, the majority of the adults and children adjusted the pendulum period depending on the length of the pendulum only. In the imagery task, many children had difficulties accurately estimating the swinging duration when imagining the pendulum swings. Adults, however, performed equally well in the imagery task and the perception task. As in the perception task, erroneous beliefs about the role of the pendulum mass did not mislead them in the imagery task. Moreover, imagery performance was not affected by the preceding task, i.e., whether the imagery task was subsequent to either the perception or the judgment task. These results indicate that, at least in terms of adults' performance, imagery was not influenced by their beliefs. In the following, we take a closer look at children and adults' performance in the three different tasks, before discussing the absolute accuracy of the answers.

\section{Perception Task}

Overall, children and adults showed an adequate knowledge of the pendulum law. As in previous studies (e.g., Kaiser et al., 1985; Kim \& Spelke, 1999), we found better performance in a perception-based task than in the more abstract judgment task where reasoning processes are more likely to be activated. Although participants could easily perceive the apparent mass, this did not affect their adjustments; pendulum length predominantly determined the responses. The analyses of individual data pattern revealed that no participant considered mass alone. It seems that adjusting a pendulum's motion to look natural draws upon representations which are in general highly unaffected by beliefs and explicit knowledge. The fact that three adults integrated length and mass in this task, however, might be a sign that explicit knowledge may interfere with perceptual knowledge for some participants. This interpretation is corroborated by the fact that many adults explicitly mentioned the mass dimension in the verbal questioning after the perception task. Interestingly, in contrast to some adults, none of the children integrated mass and length in their adjustments.

\section{Judgment Task}

In the more abstract judgment task, misbeliefs about the role of the pendulum mass could be observed in children and adults' responses, in that some children focused on the pendulum mass instead of its length, and some adults integrated information about length and mass in their judgments.

The children in this experiment were about 8 to 10 years old. It seems improbable that children at that age had thought about such problems before or had talked about them with adults. Therefore, it is even more interesting that some children considered pendulum mass. However, it is not clear whether these results can be interpreted as firm beliefs, or whether the children chose to consider any one of the two factors rather arbitrarily. The equal distribution of significant length and mass effects corroborates the latter possibility. But in any case, there is a remarkable difference between children's performance in the judgment task and in the perception task. Likewise, a dissociation between the judgment and the perception task was found in adults. Here, in view of the number of times adults explicitly mentioned mass after the judgment task, assuming some kind of firm belief about the role of pendulum mass is far more justifiable. However, how can the fact that mass is considered at all be explained?

One possible explanation rests upon the notion that it is counterintuitive to think that a change of pendulum mass does not influence the period, because without mass the pendulum would not swing at all. In other words, there is an obvious causal link between mass and the swinging behavior of the pendulum. These beliefs probably result from the overgeneralization that mass not only causes the pendulum to swing, but also influences other aspects of the swinging behavior, here the swing duration. The different ways in which the mass factor was considered shows that, even though largely convinced that mass does affect the period, participants did not unanimously agree on the $\mathrm{na}$ - 
ture of that influence. In fact, both regularities - that a heavier object can be faster (stone vs. feather falling to the ground) and that a lighter object can be faster (cars vs. trucks) - can be observed in everyday life. Reasoning about dynamic processes, and about average velocity in particular, has been repeatedly found to be very difficult (Hegarty, 1992; Huber \& Krist, 2004; Huber et al., 2003; Reed, 1984; Schwartz \& Black, 1996), and therefore participants might rely on phenomenological primitives as discussed in detail by diSessa (1993).

\section{Imagery Task}

Children's performance in the imagery task was in the main very poor. Especially in the imagery condition, the children showed very low consistencies. Due to these low consistencies, and therefore low statistical power, data were hard to interpret.

These results contrast with previous findings (Estes, 1998; Kosslyn, Margolis, Barrett, Goldknopf, \& Daly, 1990; Marmor 1975, 1977), according to which children younger than seven years old are capable of motion imagery such as mental rotation. Even children younger than four years old (Rieser, Garing, \& Young, 1994) have proven to be very proficient in imagining self-relocations under favorable conditions. However, we do not mean to contend that the children were not able to imagine the pendulum swings at all. Some children's explicit statements at the end of the task, and characteristic head or eye movements that rhythmically accompanied their imagery, refute this assumption. Rather, there is reason to believe that the children had some trouble with controlling their imagery. The present imagery task was somewhat more demanding than the tasks in previous studies in that it required highly controlled, exactly timed, and repeated imagination of an object's movement. Silent counting strategies or rigid counting rhythms may have covered the effects of the manipulated factors. This would also account for the low correlations of the responses in the two blocks. Referring to Frischeisen-Köhler (1933), Bozzi (1958) assumed that in his experiments a personal rhythm might have covered the ratings about the natural period of a pendulum. Children might be more prone to fall into such a personal counting rhythm than adults. Also, children are probably more likely to change their counting strategies during the experiment, which would also explain the low consistencies in children's responses.

Adults showed equally good performance in the imagery and perception tasks. All but one adult focused on the pendulum length. The adults' misbeliefs about pendulum mass found in the judgment task did not show in the imagery task. If the mental simulations were influenced by conceptual knowledge, then conceptual misbe- liefs would very likely have influenced performance in the imagery task. The present results, however, give reason to assume that imagery of the swinging pendulum is based on perceptual competencies rather than conceptual knowledge.

The fact that some adults integrated mass and length in the perception task but not in the imagery task might be interpreted as a sign that there is more free capacity to think about formal rules in the perception task than in the imagery task. The imagery task probably required more focused attention. Then again, it might be argued that if imagery of the swinging requires a lot of attention, it might exceed processing capacity to consider both length and mass in the imagery process. If this were the case, length would be more likely to be considered than mass. When participants look at the static pendulum and imagine the lower end of the pendulum swinging back and forth, the longer pendulum describes a longer path, which might take more time to imagine. However, Pittenger (1990) varied swing arc in one of his experiments, which did not influence participants' accuracy of detection of deviation from natural period. In his experiment, participants seemed to use period rather than angular or linear velocity as the physical variable corresponding to the speed of pendulum motion. This need not necessarily hold true for imagery as well. But the hypothesis that adults can not integrate two dimensions during imagery and therefore focus on length only is unlikely. Previous findings have shown that adults are able to integrate body-mass into their imagery of walking different distances (Decety, Jeannerod, \& Prablanc, 1989). Additional findings have shown that when adults are instructed to mentally imagine themselves walking through gates of different widths at various distances, their response times show a combined effect of gate width and distance (Decety \& Jeannerod, 1996). Furthermore, when instructed to imagine tilting a glass of water so that the water in the imagined glass reaches the rim, adults are able to integrate glass diameter and height of water level (Schwartz \& Black, 1999). The notion of a fundamental constraint to integrate two dimensions in an imagery task is refuted by these findings.

The previously completed task did not seem to affect adults' responses in the imagery task in part 2; neither the integration of the available information nor the consistencies of the responses were affected. Thus, imagery in our task seems to be quite unaffected by the knowledge context activated previously. This result contrasts with findings of Schwartz (1999), according to which a preceding judgment task interfered with the following imagery task. Schwartz's judgment task, however, required forced-choice verbal answers and so is not entirely comparable to our nonverbal and quantitative judgment task.

In conclusion, at least for adults, imagery can be a valu- 
able source of knowledge, and is quite unaffected by false beliefs. In this regard, it would be worthwhile investigating older children's use of imagery and to find out at what age and under which conditions children's performance improves.

\section{Absolute Accuracy}

No statistically significant differences could be found in the accuracy of the responses, either between the perception and the imagery task, or between the two age groups. This is quite surprising in view of the considerable differences in the participants' mean consistencies.

In the perception task, for a pendulum motion to "appear natural", the adults chose a period that was on average 0.35 seconds ( $16 \%$ of the average period) too long. This error is in line with previous findings that a pendulum moving at its natural speed seems to move too fast. Pittenger (1990), for example, concluded from his results that a pendulum moving 0.33 seconds too slow would be rated as natural. With Pittenger's average pendulum length of $106 \mathrm{~cm}$, these 0.33 seconds amounted to a mean constant error of $16 \%$. This period overestimation bias was replicated in the present experiment's perception task. Thus, the size of the mean error was comparable to results from studies that were conducted in real environments, despite the fact that virtual and scaled (1:28) stimuli were used in the present experiment, and relatively little information about the proportions was available. Though not statistically significant, this bias was reduced in the imagery task, and additionally children showed on average slightly less overestimation than adults. This tendency might be worth studying in more detail as it suggests that imagery might be less affected by perception biases and that the equivalence of imagery and perception might not comprise perceptual biases.

\section{Conclusions}

The present experiment has shown that there are differences in children and adults' period judgments, depending on different task formats. Whereas in the more abstract judgment task misbeliefs about the role of the pendulum mass could be observed with children and adults, in the more intuitive perception task virtually perfect knowledge was shown. In this intuitive task, children performed equally well as adults, even though they did not yet have formal education about the specific problem at school.

Participants' beliefs about the role of the pendulum mass, which showed in the abstract judgment task, did not influence performance in the imagery task. In our task, imagery has proven to be very robust, as no effects of the previously activated context (judgment or perception) were found. Adults' responses in the imagery task were similar to their responses in the perception task. This corroborates the assumption of the existence of informationprocessing mechanisms that are shared by both visual imagery and perception and that are not influenced by how events might be conceptualized.

\section{Author Note}

Horst Krist is now at the University of Greifswald, Germany, Department of Psychology.

\section{References}

Anderson, N. H. (1980). Information integration theory in developmental psychology. In F. Wilkening, J. Becker, \& T. Trabasso (Eds.), Information integration by children (pp. 145). Hillsdale, NJ: Lawrence Erlbaum.

Anderson, N. H. (1981). Foundations of information integration theory. New York: Academic Press.

Anderson, N. H. (1982). Methods of information integration theory. New York: Academic Press.

Bozzi, P. (1958). Analisi fenomenologica del moto pendolare armonica [Phenomenological analysis of pendular harmonic motion]. Rivista di Psychologia, 52, 281-302.

Clement, J. (1982). Students' preconceptions in introductory mechanisms. American Journal of Physics, 50, 66-71.

Decety, J., \& Jeannerod, M. (1996). Mentally simulated movements in virtual reality: Does Fitts's law hold in motor imagery? Behavioural Brain Research, 72, 127-134.

Decety, J., Jeannerod, M., \& Prablanc, C. (1989). The timing of mentally represented actions. Behavioural Brain Research, $34,35-42$.

diSessa, A. A. (1993). Toward an epistemology of physics. Cognition and Instruction, 10, 105-225.

Estes, D. (1998). Young children's awareness of their mental activity: The case of mental rotation. Child Development, 69, 1345-1360.

Finke, R. A. (1979). The functional equivalence of mental images and error of movement. Cognitive Psychology, 11, 235264.

Finke, R. A. (1980). Levels of equivalence in imagery perception. Psychological Review, 87, 113-132.

Frischeisen-Köhler, I. (1933). Das persönliche Tempo [The personal tempo]. Leipzig: Thieme.

Gilden, D., Blake, R., \& Hurst, G. (1995). Neural adaptation of imaginary visual motion. Cognitive Psychology, 28, 1-16.

Hecht, H., Kaiser, M. K., \& Banks, M. S. (1996). Gravitational acceleration as a cue for absolute size and distance? Perception \& Psychophysics, 58, 1066-1075.

Hegarty, M. (1992). Mental animation: Inferring motion from static displays of mechanical systems. Journal of Experimental Psychology: Learning, Memory, and Cognition, 18, 1084-1102. 
Huber, S., \& Krist, H. (2004). When is the ball going to hit the ground? Duration estimates, eye movements, and mental imagery of object motion. Journal of Experimental Psychology: Human Perception and Performance, 30, 431-444.

Huber, S., Krist, H., \& Wilkening, F. (2003). Judgment and action knowledge in speed adjustment tasks: Experiments in a virtual environment. Developmental Science, 6, 199-212.

Johansson, G., \& Jansson, G. (1967). The perception of free fall. Unpublished seminar paper, Department of Psychology, University of Uppsala, Sweden.

Kaiser, M. K., \& Proffitt, D. R. (1984). The development of sensitivity to causally relevant dynamic information. Child Development, 55, 1614-1624.

Kaiser, M. K., Proffitt, D. R., \& Anderson, K. (1985). Judgments of natural and anomalous trajectories in the presence and absence of motion. Journal of Experimental Psychology: Learning, Memory, and Cognition, 11, 795-803.

Kim, In-Kyeong, \& Spelke, E. S. (1999). Perception and understanding of effects of gravity and inertia in object motion. Developmental Science, 2, 339-362.

Kosslyn, S. M., Margolis, J. A., Barrett, A. M., Goldknopf, E. J., \& Daly, P.F. (1990). Age differences in imagery ability. Child Development, 61, 995-1010.

Kosslyn, S. M., Thompson, W. L., Kim, I. J., \& Alpert, N. M. (1995). Topographical representations of mental images in primary visual cortex. Nature, 378, 496-498.

Krist, H., Fieberg, E. L., \& Wilkening, F. (1993). Intuitive physics in action and judgment: The development of knowledge about projectile motion. Journal of Experimental Psychology: Learning, Memory, and Cognition, 19, 952-966.

Krist, H., Loskill, J., \& Schwarz, S. (1996). Intuitive Physik in der Handlung: Perzeptiv-motorisches Wissen über Flugbahnen bei 5-7-jährigen Kindern [Intuitive physics in action: Perceptual-motor knowledge about projectile motion in 5-7year-old children]. Zeitschrift für Psychologie, 204, 339366.

McCloskey, M. (1983). Intuitive physics. Scientific American, 248, 122-130.

McCloskey, M., Caramazza, A., \& Green, B. (1980). Curvilinear motion in the absence of external forces: Naive beliefs about the motion of objects. Science, 210, 1139-1141.

Marmor, G. S. (1975). Development of kinetic images: When does the child first represent movement in mental images? Cognitive Psychology, 7, 548-559.

Marmor, G. S. (1977). Mental rotation and number conservation: Are they related? Developmental Psychology, 13, 320325.

Piaget, J. (1970). Piaget's theory. In P. H. Mussen (Ed.), Carmichael's manual of child psychology (Vol. 1). New York: Wiley.

Pittenger, J. B. (1985). Estimation of pendulum length form information in motion. Perception, 14, 247-256.

Pittenger, J. B. (1990). Detection of violations of the law of pendulum motion: Observers' sensitivity to the relation between period and length. Ecological Psychology, 2, 55-81.
Reed, S. K. (1984). Estimating answers to algebra word problems. Journal of Experimental Psychology: Learning, Memory, and Cognition, 10, 778-790.

Rieser, J. J., Garing, A. E., \& Young, M. F. (1994). Imagery, action, and young children's spatial orientation: It's not being there that counts, it's what one has in mind. Child Development, 65, 1262-1278.

Schwartz, D. L. (1999). Physical imagery: Kinematic versus dynamic models. Cognitive Psychology, 38, 433-464.

Schwartz, D. L., \& Black, J. B. (1996). Analog imagery in mental model reasoning: Depictive models. Cognitive Psychology, 30, 154-219.

Schwartz, D. L., \& Black, T. (1999). Inferences through imagined actions: Knowing by simulated doing. Journal of Experimental Psychology: Learning, Memory, and Cognition, $25,116-136$.

Shanon, B. (1976). Aristotelianism, Newtonianism and the physics of the layman. Perception, 5, 241-243.

Spelke, E. S., Breinlinger, K. Macomber, J., \& Jacobson, K. (1992). Origins of knowledge. Psychological Review, 99, 605-632.

Spillane, C., Turcotte, T., \& Pufall, P. B. (1991). Predicting pendulum length from motion: A developmental study. In P. J. Beek, R. J. Bootsma, \& P. C. W. van Wieringen (Eds.), Studies in perception and action. Posters presented at the VIth International Conference on Event Perception and Action. Amsterdam: Radopi.

Watson, J. S., Banks, M. S., von Hofsten, C., \& Royden, C. S. (1992). Gravity as a monocular cue for perception of absolute distance and/or absolute size. Perception, 21, 69-76.

Wilkening, F. (1980). Development of dimensional integration in children's perceptual judgment: Experiments with area, volume, and velocity. In F. Wilkening, J. Becker, \& T. Trabasso (Eds.), Information Integration by children (pp. 4769). Hillsdale, NJ: Lawrence Erlbaum.

Wilkening, F. (1981). Integrating velocity, time and distance information: A developmental study. Cognitive Psychology, $13,231-247$.

Wilkening, F., \& Huber, S. (2002). Children's intuitive physics. In U. Goswami (Ed.), Blackwell handbook of childhood cognitive development (pp. 349-370). Oxford: Blackwell.

Andrea Frick

Department of Psychology

University of Zürich

Attenhoferstraße 9

CH-8032 Zürich

Switzerland

a.frick@psychologie.unizh.ch 